\section{MS19-P7 Sn/C composite anode materials for high energy lithium/sodium ion batteries}

Sivarajakumar Maharajan $^{1}$, Nam Hee Kwon ${ }^{1}$, Katharina M. Fromm ${ }^{1}$

\section{University of Fribourg, Switzerland}

email: sivarajakumar.maharajan@unifr.ch

\section{Introduction:}

Metallic tin ( $\mathrm{Sn}$ ) is one of the most promising anode materials for next-generation LIBs due to its high theoretical capacity of $991 \mathrm{mAhg}^{-1}$ or $7313 \mathrm{mAhcm}^{-3}$, multiple times that of commercialized graphite anode materials $\left(372 \mathrm{mAhg}^{-1}\right.$ or $\left.833 \mathrm{mAhcm}^{-3}\right)$. However, the huge volume expansion (up to $360 \%$ ) consequently leading to the dramatic mechanical stress of Sn during cycling cause cracking and pulverization of the active material (refer Fig. 1). This also leads to the loss of conductivity at the electrode, resulting in quick capacity fading, which greatly hinders the practical application of $\mathrm{Sn}$ as anode material and thereby letting down its application in $\operatorname{LIBs}^{[2,3]}$. It is therefore essential to control the volume expansion and thereby preventing the loss of active materials. A composite electrode which could facilitate a buffer volume to survive the volume expansion will be the call of the moment. We have adopted a novel synthesis method to form a nano-rattle composite anode made of Sn nano metal encapsulated into carbon shell as shown in figure 1 .

Reverse micelle micro emulsion synthesis:

A reverse micelle micro emulsion technique has been developed to form Sn encapsulated into silica shell where silica shell serves as substrate for carbon coating[4]. A non-structured carbon coating has been formed on silica shell. To enhance the conductivity and mechanical strength, the obtained $\mathrm{Sn} @ \mathrm{SiO}_{2} @ \mathrm{C}$ has been further heated to $700^{\circ} \mathrm{C}$ under Argon to obtain a well-structured carbon coated $\mathrm{Sn} @ \mathrm{SiO}$. Further etching of $\mathrm{SiO}_{2}$ layer by treating with $10 \% \mathrm{HF}$ creates a void between $\mathrm{Sn}$ nanoparticle and carbon shell, yielding Sn@C nano-rattles. Figure 3 shows the TEM picture showing Sn encapsulated into carbon shell with void in between. Also, the cyclic voltammetry shows the redox behaviour of $\mathrm{Sn}$ and $\mathrm{SiO}_{2}$.

Conclusion:

$\mathrm{Sn} / \mathrm{C}$ nano-rattle composite anode material has been synthesised. However, the $\mathrm{SiO}_{2}$ removal is incomplete which hinders the redox behaviour contribution of $\mathrm{Sn}$. Successful removal of $\mathrm{SiO}_{2}$ completely will be followed by making electrodes and assembling of half cells.

References:

[1] W.-J. Zhang, Journal of Power Sources 2011, 196, 13-24.

[2] J. O. Besenhard, J. Yang and M. Winter, Journal of Power Sources 1997, 68, 87-90.

[3] D. Aurbach, Journal of Power Sources 2000, 89, 206-218.

[4] M. Priebe and K. M. Fromm, Particle \& Particle Systems Characterization 2014, 31, 645-651.
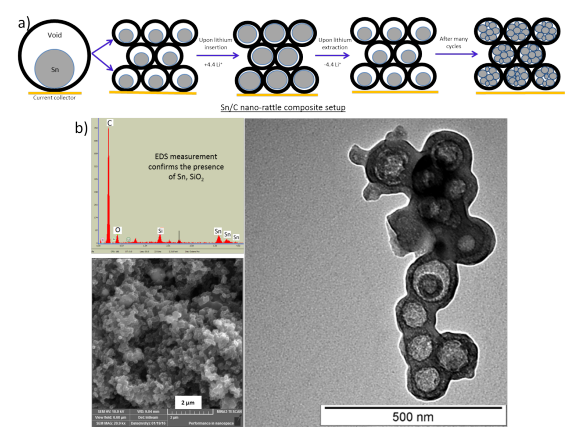

Figure 1. Sn/C nano-rattle set up to avoid volume expansion (a), SEM/SEM images with EDS measurement

Keywords: lithium ion batteries, sodium ion batteries, alloy based anode material 